[4] G. E. Backus and J. F. Gilbert, "Numerical applications of a formalism for geophysical inverse problems," Geophys. J. Roy. Astron. Soc., vol. 13, pp. 247-276, 1967.

[5] K. Sekihara and B. Scholz, "Generalized wiener estimation of three dimensional current distribution from biomagnetic measurements," IEEE Trans. Biomed. Eng., vol. 43, pp. 281-291, Mar. 1996.

[6] R. G. de Peralta Menendez, O. Hauk, S. L. G. Andino, H. Vogt, and C. M. Michel, "Linear inverse solutions with optimal resolution kernels applied to the electromagnetic tomography," Human Brain Mapping, vol. 5, pp. 454-467, 1997.

[7] R. G. de Peralta Menendez and S. L. G. Andino, "Discussing the capabilities of Laplacian minimization," Brain Topogr., vol. 13, pp. 97-104, 2000.

[8] R. G. de Peralta Menendez, S. L. G. Andino, G. Lantz, C. M. Michel, and T. Landis, "Noninvasive localization of electromagnetic epileptic activity. I method descriptions and simulations," Brain Topogr., vol. 14, pp. 131-137, 2001.

[9] J. P. Ary, S. A. Klein, and D. H. Fenders, "Location of sources of evoked scalp potentials: Correction for skull and scalp thicknesses," IEEE Trans. Biomed. Eng., vol. BME-28, pp. 447-452, 1981.

[10] R. G. de Peralta Menendez and S. L. G. Andino. (2002) Comparison of algorithms for the localization of focal sources: Evaluation with simulated data and analysis of experimental data. Int. J. Bioelectromagn. [Online]. Available: http://www.ee.tut.fi/rgi/ijbem/volume4/number1/toc.htm

[11] A. R. Davies, Optimality in Regularization. In Inverse Problems in Scattering and Imaging, M. Bertero and E. R. Pike, Eds. New York: Adam Hilger, 1992.

\section{Role of Transdermal Potential Difference During Iontophoretic Drug Delivery}

\author{
Andriy Bandrivskyy, Alan Bernjak, Peter V. E. McClintock, and \\ Aneta Stefanovska*
}

\begin{abstract}
Potential differences have been measured during transdermal iontophoresis in order to establish the effect of voltage, as opposed to current, on cutaneous blood flow. It is known that, even in the absence of drugs, the iontophoresis current can sometimes produce increased blood flow. The role of voltage in this process is studied through single-ended measurements (between electrode and body) of the potential difference during iontophoresis with $100-\mu \mathrm{A}, 20$-s current pulses through deionized water, saturated $20.4 \% \mathrm{NaCl}$ solution, $1 \%$ acetylcholine, and $1 \%$ sodium nitroprusside. It is found that the voltage needed to deliver the current varied by orders of magnitudes less than the differences in the conductance of these different electrolytes, and it is concluded that, at least for the present current protocol, the voltage as such is not an important factor in increasing the blood flow.
\end{abstract}

Index Terms-Blood flow, iontophoresis, laser-doppler flowmetry, transdermal potential.

Manuscript received August 20, 2003; revised February 8, 2004. This work was supported by the Engineering and Physical Sciences Research Council (U.K.), the Joy Welch Trust (U.K.), INTAS, the British Council, and the Slovenian Ministry of Education, Science and Sport. Asterisk indicates corresponding author.

A. Bandrivskyy and P. V. E. McClintock are with the Department of Physics, Lancaster University, Lancaster LA1 4YB, U.K.

A. Bernjak is with the Nonlinear Dynamics and Synergetics Group, Faculty of Electrical Engineering, University of Ljubljana, Tržaška 25, 1000 Ljubljana, Slovenia.

*A. Stefanovska is with the Group of Nonlinear Dynamics and Synergetics, Faculty of Electrical Engineering, University of Ljubljana, Tržaška 25, 1000 Ljubljana, Slovenia and the Department of Physics, Lancaster University, Lancaster LA1 4YB, U.K.

Digital Object Identifier 10.1109/TBME.2004.828039
TABLE I

Summary of Data for the 11 Subjects, Showing Their Gender, Age, and the AVERage Potential DifFerences (IN Volts) NEEDED to DELIVER IONTOPHORESIS CURRENTS OF $100 \mu \mathrm{A}$, FOR THE SIX Electrolyte Configurations. In EACH Data-SET THE MEDian VALUe Is EMBOLDENED

\begin{tabular}{c|r|rrrrrr}
\hline Subject & $\begin{array}{r}\text { gender } \\
\text { \& age }\end{array}$ & $\begin{array}{r}\mathrm{H}_{2} \mathrm{O} \\
(-)\end{array}$ & $\begin{array}{r}\mathrm{H}_{2} \mathrm{O} \\
(+)\end{array}$ & $\begin{array}{r}\mathrm{NaCl} \\
(-)\end{array}$ & $\begin{array}{r}\mathrm{NaCl} \\
(+)\end{array}$ & $\begin{array}{r}\mathrm{SNP} \\
(-)\end{array}$ & $\begin{array}{r}\text { ACh } \\
(+)\end{array}$ \\
\hline $\mathrm{A}$ & $\mathrm{f}, 24$ & $\mathbf{1 2 . 5}$ & 13.8 & 11.1 & 12.1 & 12.2 & $\mathbf{1 3 . 5}$ \\
$\mathrm{B}$ & $\mathrm{m}, 24$ & 8.2 & 10.6 & 7.6 & 10.0 & 10.0 & 11.6 \\
$\mathrm{C}$ & $\mathrm{m}, 24$ & 19.9 & 21.3 & 12.8 & 15.5 & 16.6 & 13.8 \\
$\mathrm{D}$ & $\mathrm{m}, 26$ & 14.0 & 16.4 & 11.2 & 12.7 & 11.0 & 14.3 \\
$\mathrm{E}$ & $\mathrm{m}, 35$ & 11.3 & $\mathbf{1 5 . 0}$ & 4.2 & 5.9 & 10.7 & 14.3 \\
$\mathrm{~F}$ & $\mathrm{f}, 44$ & 13.0 & 11.9 & $\mathbf{9 . 7}$ & $\mathbf{1 1 . 1}$ & $\mathbf{9 . 5}$ & 12.8 \\
$\mathrm{G}$ & $\mathrm{m}, 45$ & 6.7 & 9.7 & 4.8 & 3.1 & 6.5 & 10.7 \\
$\mathrm{H}$ & $\mathrm{m}, 51$ & 16.2 & 18.0 & 14.2 & 17.5 & 13.1 & 15.3 \\
$\mathrm{I}$ & $\mathrm{m}, 51$ & 8.0 & 15.6 & 10.5 & 7.8 & 7.0 & 7.5 \\
$\mathrm{~J}$ & $\mathrm{~m}, 62$ & 10.9 & 12.4 & 9.4 & 10.4 & 5.6 & 10.7 \\
$\mathrm{~K}$ & $\mathrm{~m}, 68$ & 17.1 & 16.8 & 9.5 & 14.6 & 8.5 & 14.1 \\
\hline
\end{tabular}

\section{INTRODUCTION}

Iontophoresis is widely used for transcutaneous delivery of ionizable drugs for the assessment of skin microvascular function. In practice, a small electrical current is used to carry e.g., vasodilators such as acetylcholine (ACh) and sodium nitroprusside (SNP) through the skin, while consequent changes in blood flow are monitored by laser Doppler flowmetry [1]-[3]. It is known, however, that increases of blood flow can be produced in response to the current, even in the absence of drugs, when using pharmacologically neutral electrolytes such as $\mathrm{H}_{2} \mathrm{O}$ [4], [5] or $\mathrm{NaCl}$ solution [6]. This phenomenon is known as the galvanic effect or current-induced vasodilation. The mechanism is unclear but could involve, for example, local heating due to the voltage required to convey the ions through the dermal barrier. Because a finite potential difference is always needed to maintain the chosen iontophoresis current, it is obviously important to establish whether or not the magnitude of this voltage is a significant factor in causing vasodilation.

We have therefore carried out a systematic study of the voltages needed for iontophoresis with a constant current under different conditions, and of how they change with time. The present work differs in two important respects from the investigations recently reported by Ferrell et al. [7] and Ramsay et al. [8]. First, all of our measurements are for pulsed iontophoresis, rather than for a continuous longer period of stepped current delivery. Second, our measurements are all single-ended, i.e., we measure the potential difference when a fixed current is passed between an iontophoresis cell and the body, rather than the combined voltage difference across two cells of opposite polarity, with the body in between. This enables us to establish unambiguously the voltage needed for iontophoresis of given polarity with particular electrolytes.

\section{MethodS}

The measurements were performed on 11 healthy subjects (two female, nine male) aged 24-68 years (see Table I). They lay comfortably, semi-supine, with the measured forearm placed in a horizontal position on a cushion, in a quiet room with an ambient temperature of $20 \pm 1^{\circ} \mathrm{C}$. The study was approved by the Morecambe Bay Local Research Ethics Committee and the subjects gave their fully informed consent.

Currents were derived from a battery-powered constant-current iontophoresis controller (Moor Instruments MIC1-e). The iontophoresis chambers were of perspex with internal platinum wire electrodes. Their internal diameter was $8 \mathrm{~mm}$, giving an area of $1 \mathrm{~cm}^{2}$ in contact with the skin. Four chambers were used, placed in a square of side $2 \mathrm{~cm}$ on the volar aspect of the forearm. They were held in position with doublesided adhesive disks, avoiding hair or skin defects. The earth-potential electrode was a $4 \mathrm{~cm} \times 4 \mathrm{~cm}$ conducting pad, placed on the wrist at 

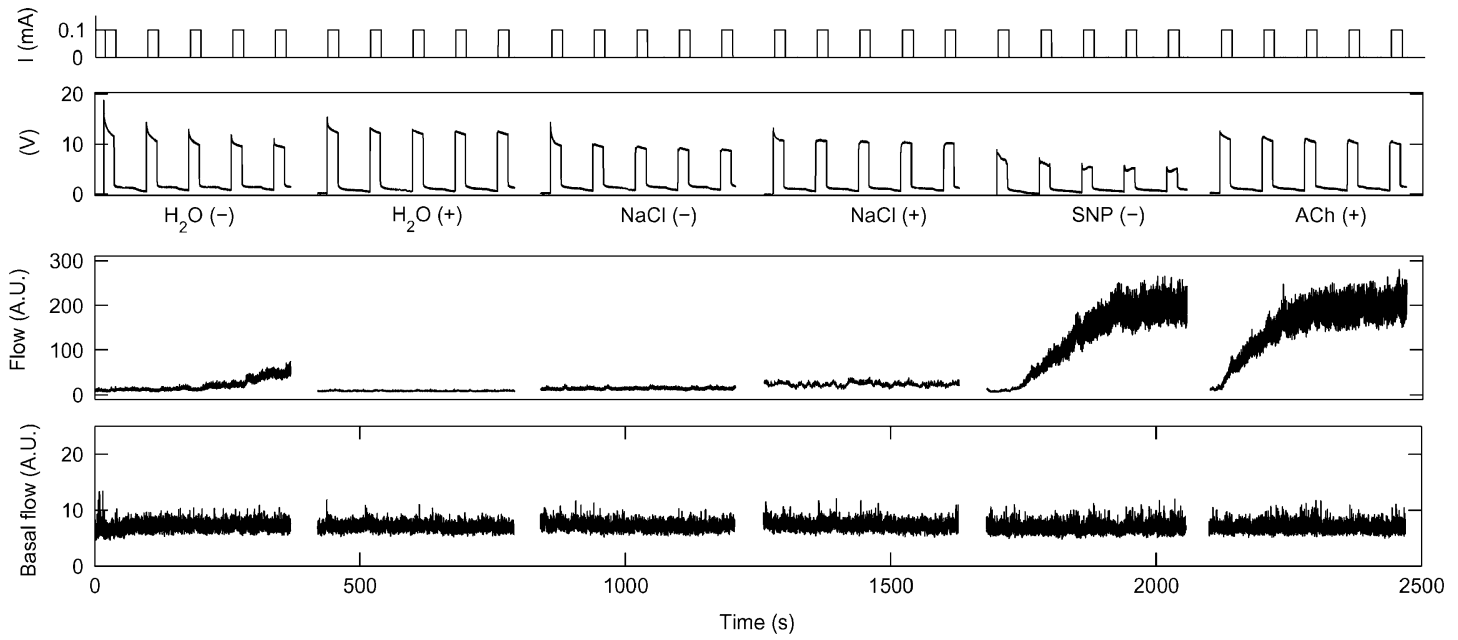

Fig. 1. Potential differences (in volts) needed to drive the iontophoretic current pulses (see Section II), for subject J. The top panel indicates the timing of the current pulses. The lower panels show respectively: voltage as a function of time for the different electrolytes and polarities of active electrode, as indicated; blood flow measured by the probe inserted in the centre of the chamber; and basal blood flow in unstimulated skin at least $5 \mathrm{~cm}$ away from the nearest iontophoresis chamber. Note the difference in ordinate scale between the lower two panels. The abscissa scale is the same for all measurements and electrolytes, but with an arbitrary zero and different offsets for the different electrolytes.

a distance of $15-18 \mathrm{~cm}$ from the iontophoresis chamber. Good electrical contact was ensured by the use of conducting MIC1-GP gel. The four substances were inserted sequentially, and randomly. The order in which the polarities were chosen was also random. For consistency, the discussion that follows relates to the order of the data in Fig. 1, but this does not reflect the order in which the data were acquired.

The voltage required to maintain a constant current between each chamber and the earth-potential electrode was measured. The voltage measurement circuit was of very high input impedance $(50 \mathrm{M} \Omega$ ) to avoid drawing a significant fraction of the current from the iontophoresis controller. It was based on the Linear Technology LT1112 operational amplifier for the input stages, and a Burr Brown 3656 transformer-coupled isolation amplifier meeting the safety requirements of IEC 601-1. The circuit also served to rescale the measured \pm 25 to $\pm 5 \mathrm{~V}$, which was taken to the same multi-channel analogue-to-digital convertor used for digitizing the blood flow signals.

The programmed current delivery protocol produced $100-\mu \mathrm{A}$ pulses of duration $20 \mathrm{~s}$, separated by 60 -s intervals, over a total measurement period of $6 \mathrm{~min}$. Between the pulses, the iontophoresis current was held at zero.

Voltage measurements were made for four different electrolytes: deionized $\mathrm{H}_{2} \mathrm{O} ; 20.4 \%$ saturated, $5 \mathrm{~mol} / \mathrm{l} \mathrm{NaCl}$ solution (both positive and negative electrodes); $1 \%$ ACh (positive electrode); and 1\% SNP (negative electrode). During these measurements, the cutaneous blood flow was monitored by laser/Doppler flowmetry using the Moor Instruments DRT4 to which the iontophoresis controller was attached.

\section{RESULTS}

A full set of iontophoresis voltage measurements for subject $\mathrm{J}$ is shown in Fig. 1. In what follows, we use average to denote a time average of the blood flow or of the potential difference, while median refers to the group median value over the 11 subjects. The average voltages for each subject are summarized in Table I and presented graphically in Fig. 2(a). Several features of the data are apparent. The average magnitude of the voltage needed to maintain the current decreased with time: each successive pulse is slightly lower. The voltages also varied within each pulse, usually decreasing as shown in Fig. 1. The voltage between current pulses was in general small but nonzero, and it sometimes varied with time in a complicated manner.
Cathodal iontophoresis with deionized $\mathrm{H}_{2} \mathrm{O}$ produced clear evidence of increased blood flow, as shown in Fig. 2(b); but even in the seven subjects where the effect was significant, the increase was smaller by a factor of $\sim 4$ than that seen in iontophoresis with either of the vasodilators ACh or SNP. The average blood flows, stimulated and basal values, for all subjects and six configurations are shown in Fig. 2(b).

The averaged blood flow measured in each of the subjects is shown for different forms of iontophoresis in Table II. Statistical analyses were performed to assess the possibility of correlations between voltage and blood flow. Although 11 subjects is hardly a sufficient number for serious correlation analysis, it may be of interest that we did not observe any statistically significant correlation for any mode of iontophoresis. The analyses were repeated for each individual, while considering the six modes of iontophoresis, again with a null result in each case.

The polarity that produced greater voltage was associated with a smaller blood flow when deionized $\mathrm{H}_{2} \mathrm{O}$ was used. Cathodal iontophoresis resulted in increased average blood flow, although not significantly greater than the blood flow obtained during anodal current $(p=0.1)$. At the same time, the average voltage was slightly greater during anodal iontophoresis but, again, not significantly $(p=0.3)$. When the group values are compared for the $\mathrm{NaCl}$, it can be seen that a slightly higher, but not significant $(p=0.4)$, average potential difference is obtained during anodal iontophoresis, while the average blood flow values were comparable. Although the voltage was significantly higher during ACh compared to SNP iontophoresis $(p<0.05)$, this difference was not reflected in the average blood flow values $(p=0.4)$. Average blood flow values during iontophoresis with ACh are slightly higher, but not significantly $(p=0.4)$, than those obtained with SNP.

\section{DISCUSSION}

The results obtained appear to be consistent with earlier work [7], [8], although the different current delivery protocol used in the latter, and the fact that the individual voltages needed to deliver particular substances could not be separated, makes direct comparison difficult.

The group median values of average voltages during iontophoresis with deionized $\mathrm{H}_{2} \mathrm{O}$ are $15.0 \mathrm{~V}$ for anodal current and $12.5 \mathrm{~V}$ for cathodal current. These values are slightly higher than the median values of voltages during iontophoresis with saturated $\mathrm{NaCl}$, which are $11.1 \mathrm{~V}$ for anodal and $9.7 \mathrm{~V}$ for cathodal currents. It seems, at first 

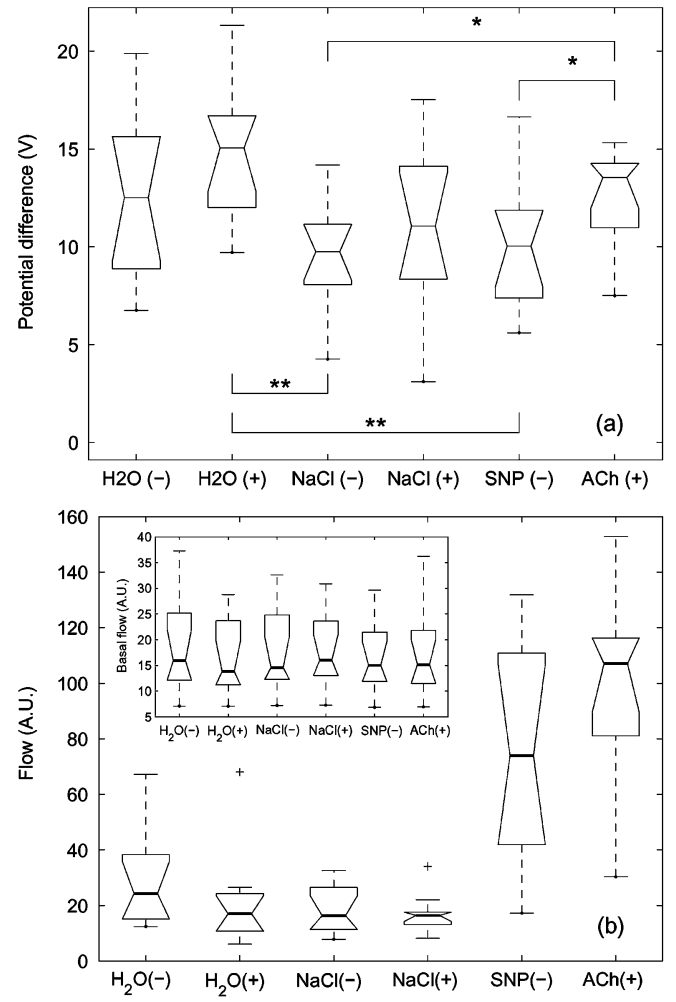

Fig. 2. (a) Box and whisker plots of average potential difference and (b) average blood flow values as functions of substance and current polarity used for iontophoresis in a group of 11 healthy subjects. The inset in (b) shows average values of basal blood flow measured simultaneously in unstimulated skin several $\mathrm{cm}$ from the iontophoresis chambers. The boxes have lines at the lower quartile, median and upper quartile values. The whiskers extend from each end of the box and their maximal length is set to 1.5 times the size of the box. Outlier values are presented as + . The probability that the potential difference is the same for all tested substances and polarities, evaluated by ANOVA, is 0.009 . This means that out of six data sets, at least one is significantly different from the others. The probability obtained by nonparametric, unpaired Wilcoxon test from a pair of data sets is marked as* if $0.05>p>0.01$ and ${ }^{* *}$ for $p<0.01$. The overall difference between average blood flow values obtained in six different measurements, tested by ANOVA, is highly significant $(p=0)$. The average blood flow values obtained during iontophoresis with ACh or SNP differ significantly $(p<0.05)$ from those obtained during iontophoresis with $\mathrm{H}_{2} \mathrm{O}$ and $\mathrm{NaCl}$ using both polarities, but there is no significant difference between the flows during ACh and SNP $(p=0.4)$. On the other hand, the basal blood flow values do not differ significantly for any of the six recordings.

TABLE II

SUMMARY OF DATA FOR THE 11 SUBJECTS, SHOWING THEIR AVERAGED BLOOD FLOW (ARBITRARY UNITS) DURING DIFFERENT FORMS OF IONTOPHORESIS. IN EACH DATA-SET THE MEDIAN VALUE Is EMBOLDENED

\begin{tabular}{c|rrrrrr}
\hline Subject & $\begin{array}{r}\mathrm{H}_{2} \mathrm{O} \\
(-)\end{array}$ & $\begin{array}{r}\mathrm{H}_{2} \mathrm{O} \\
(+)\end{array}$ & $\begin{array}{r}\mathrm{NaCl} \\
(-)\end{array}$ & $\begin{array}{r}\mathrm{NaCl} \\
(+)\end{array}$ & $\begin{array}{r}\mathrm{SNP} \\
(-)\end{array}$ & $\begin{array}{r}\text { ACh } \\
(+)\end{array}$ \\
\hline $\mathrm{A}$ & $\mathbf{2 7 . 3}$ & 68.0 & 7.8 & 15.5 & 17.3 & 136.8 \\
$\mathrm{~B}$ & 15.7 & 24.8 & 14.1 & 17.1 & 55.1 & 81.1 \\
$\mathrm{C}$ & 41.0 & 19.6 & 22.1 & 22.0 & 18.3 & 9.1 \\
$\mathrm{D}$ & 38.3 & 10.6 & 32.6 & 8.2 & 87.9 & 71.2 \\
$\mathrm{E}$ & 35.3 & $\mathbf{1 7 . 0}$ & $\mathbf{1 6 . 3}$ & 11.1 & 87.2 & $\mathbf{1 0 5 . 9}$ \\
$\mathrm{F}$ & 12.4 & 9.5 & 26.5 & $\mathbf{1 6 . 4}$ & 131.7 & 97.0 \\
$\mathrm{G}$ & 155.4 & 26.5 & 26.5 & 17.5 & 37.5 & 116.3 \\
$\mathrm{H}$ & 13.5 & 22.8 & 27.5 & 12.5 & $\mathbf{7 3 . 9}$ & 30.3 \\
$\mathrm{I}$ & 15.2 & 11.12 & 11.2 & 14.9 & 71.45 & 108.2 \\
$\mathrm{~J}$ & 21.3 & 6.0 & 8.2 & 34.0 & 123.3 & 152.8 \\
K & 67.2 & 14.2 & 12.9 & 17.6 & 118.5 & 115.5 \\
\hline
\end{tabular}

sight, surprising that the measured voltages should all fall within a range that is so much narrower than the enormous range in the conductance of the two electrolytes. At the two extremes, $\mathrm{NaCl}$ solution is of very low resistance, whereas deionized $\mathrm{H}_{2} \mathrm{O}$ is in principle an insulator. Using exactly the same current delivery protocol, but with the electrodes immersed in bulk electrolyte in a Petri dish:

1) for deionized $\mathrm{H}_{2} \mathrm{O}$, the Moor iontophoresis controller was able to deliver only $25-28 \mu \mathrm{A}$, rather than the chosen $100 \mu \mathrm{A}$, and the voltage was out of range;

2) with $\mathrm{NaCl}$ solution, the voltage was too small to measure reliably, being only about twice the background noise level.

This behavior is exactly what was to be expected, given the difference of many orders of magnitude in the relative conductances. The fact that (initially) deionized $\mathrm{H}_{2} \mathrm{O}$ does not behave as a near-insulator in iontophoresis through skin is presumably because ions are quickly acquired from/through the skin itself.

The relatively small range of voltages, and the fact that they vary systematically between individuals (Table I) suggest that, at least for the current protocol used in the present study, the conductance of the electrolyte is relatively unimportant. The main contribution to the resistance is probably that of the transdermal barrier.

Our statistical analyses revealed no correlation between voltage and blood flow. It would seem, therefore, that the changes in blood flow that are sometimes observed in the absence of vasoactive agents are not attributable to subcutaneous ohmic heating in those subjects for whom the voltage needed to drive iontophoresis is relatively large.

\section{CONCLUSION}

Although many details remain to be explored, the main question motivating this investigation can be answered unambiguously. At least for the conditions applying in the present investigation, the magnitude of the voltage needed to sustain the chosen iontophoresis current is not an important factor in causing changes in blood flow. This conclusion may be expected to apply, not only to neutral agents such as $\mathrm{H}_{2} \mathrm{O}$ or $\mathrm{NaCl}$ solution, but also in iontophoretic drug delivery.

\section{ACKNOWLEDGMENT}

The authors are grateful to all the subjects who volunteered and they acknowledge valuable discussions and help from R. Gush, S. Holt, I. Kaufman, and M. Lippett.

\section{REFERENCES}

[1] S. J. Morris and A. C. Shore, "Skin blood flow responses to the iontophoresis of acetylcholine and sodium nitroprusside in man: Possible mechanisms," J. Physiol. (London), vol. 496, pp. 531-542, 1996.

[2] H. D. Kvernmo, A. Stefanovska, K.-A. Kirkebøen, and K. Kvernebo, "Oscillations in the human cutaneous blood perfusion signal modified by endothelium-dependent and endothelium-independent vasodilators," Microvasc. Res., vol. 57, pp. 298-309, 1999.

[3] A. Stefanovska, M. Bračič, and H. D. Kvernmo, "Wavelet analysis of oscillations in the peripheral blood circulation measured by laser Doppler technique," IEEE Trans. Biomed. Eng., vol. 46, pp. 1230-1239, Oct. 1999.

[4] M. N. Berliner, "Skin microcirculation during tapwater iontophoresis in humans: Cathode stimulates more than anode," Microvasc. Res., vol. 54, pp. 74-80, 1997.

[5] S. Durand, B. Fromy, P. Bouyé, J. L. Saumet, and P. Abraham, "Currentinduced vasodilation during water iontophoresis $(5 \mathrm{~min}, 0.01 \mathrm{~mA})$ is delayed from current onset and involves aspirin-sensitive mechanisms," J. Vasc. Res., vol. 39, pp. 59-71, 2002.

[6] A. Asberg, T. Holm, T. Vasbotn, A. K. Andreassen, and A. Hartman, "Nonspecific microvascular vasodilation during iontophoresis is attenuated by application of hyperosmolar saline," Microvasc. Res., vol. 58, pp. 41-48, 1999.

[7] W. R. Ferrell, J. E. Ramsay, N. Brooks, J. C. Lockhart, S. Dickson, G. M. McNeece, I. A. Greer, and N. Sattar, "Elimination of electrically induced iontophoretic artefacts: Implications for noninvasive assessment of peripheral microvascular function," J. Vasc. Res., vol. 39, pp. 447-455, 2002.

[8] J. E. Ramsay, W. R. Ferrell, I. A. Greer, and N. Sattar, "Factors critical to iontophoretic assessment of vascular reactivity: Implications for clinical studies of endothelial disfunction," J. Cardiovasc. Pharmacol., vol. 39, pp. $9-17,2002$. 